\title{
48. DATA REPORT: SULFUR CONTENT OF SEDIMENT AND SULFUR ISOTOPE VALUES OF SULFIDE AND SULFATE MINERALS FROM MIDDLE VALLEY ${ }^{1}$
}

\author{
Robert A. Zierenberg ${ }^{2}$
}

\section{INTRODUCTION}

Sulfide sulfur content of sediment and sulfur isotope ratios of sulfide and sulfate minerals were measured as part of an effort to determine the sources and mass and isotope fluxes of sulfur in and near the Middle Valley hydrothermal system. Limited access to mass spectrometry facilities resulted in the late attainment of these data relative to the deadline for submission of manuscripts to this volume. Therefore, these data are presented as a data report without significant interpretation.

\section{METHODS}

Samples for sulfur isotope analyses were classified by major lithology as sediment, diabase, basalt, massive sulfide, or clastic sulfide redeposited from massive sulfide. Sediment samples were classified as clay, silt, or sand based on a visual estimation of the dominant grain size. Total sulfide sulfur was chemically extracted from bulk samples of unlithified sediment by acid dissolution in $6 \mathrm{~N} \mathrm{HCl}$ using chromous chloride as a reductant (Canfield et al., 1986). $\mathrm{H}_{2} \mathrm{~S}$ produced in the reaction was stripped from the solution by $\mathrm{N}_{2}$ gas and precipitated as $\mathrm{Ag}_{2} \mathrm{~S}$. The weight of $\mathrm{Ag}_{2} \mathrm{~S}$ produced relative to the starting weight of sediment provides a measure of the sulfide sulfur content in the sediments (Table 1). Injection of $\mathrm{HCl}$ into the reaction vessels prior to addition of $\mathrm{CrCl}_{2}$ allowed detection and separation of acid-volatile sulfide minerals such as pyrrhotite, mackinawite, and greigite. Acid volatile sulfide was detected only in trace amounts and only in a small number of samples. The total sulfide content and the sulfur isotope data therefore reflect pyritic sulfur (Table 1).

In more lithified sediment samples and in samples of basalt, diabase, or massive sulfide, subsamples for sulfur isotope analyses were taken by drilling with diamond-impregnated bits using a handheld dental drill. This often allowed sampling of individual minerals, veins, burrow fills, or nodules of sulfide and sulfate minerals. Separate subsamples are listed on separate lines in Table 1. When more than one mineral was analyzed from the same subsample the results are listed on the same line of the table. Because of the selective sampling, sulfur contents are not reported for these samples. All sulfide samples were chemically treated to extract monosulfide sulfur (sphalerite and/or pyrrhotite), by dissolution under an $\mathrm{N}_{2}$ atmosphere in $6 \mathrm{~N} \mathrm{HCl}$, followed by extraction of disulfide sulfur (pyrite, marcasite, and chalcopyrite) using $\mathrm{CrCl}_{2}$ as a reductant. Sulfate-mineral-bearing samples were treated with $0.5 \mathrm{~N} \mathrm{HCl}$ to dissolve anhydrite and gypsum; the dissolved sulfate was reprecipitated as $\mathrm{BaSO}_{4}$. Samples with barite were first treated to remove anhydrite and gypsum followed by dissolution of barite in concentrated $\mathrm{NaCO}_{3}$ and reprecipitation as $\mathrm{BaSO}_{4}$, as described by Breit et al. (1985).

Sulfur isotope determinations were made on $\mathrm{SO}_{2}$ produced by combustion of $\mathrm{Ag}_{2} \mathrm{~S}$ under vacuum using $\mathrm{Cu}_{2} \mathrm{O}$ as an oxidant, or combustion of $\mathrm{BaSO}_{4}$ with $\mathrm{Cu}_{2} \mathrm{O}$ plus quartz. Measurements were made

\footnotetext{
${ }^{1}$ Mottl, M.J., Davis, E.E., Fisher, A.T., and Slack, J.F. (Eds.), 1994. Proc. ODP, Sci. Results, 139: College Station, TX (Ocean Drilling Program).

${ }^{2}$ U.S. Geological Survey, 345 Middlefield Rd., M.S. 901, Menlo Park, CA $94025-$ 3591, U.S.A.
}

on a Finnigan Delta mass spectrometer and standardized against $\mathrm{Ag}_{2} \mathrm{~S}$ standards with values determined by $\mathrm{SF}_{6}$ by Rees (1978). Present day seawater sulfate determined by this method gives a value of $20.99 \%$ relative to Canyon Diablo Troilite (CDT) (Rees et al., 1978).

\section{RESULTS}

\section{Site 855}

Site 855 was rotary cored in an area of low heat flow near a normal fault that bounds the east side of Middle Valley. Sulfur content of sediments recovered at this site are low ( 0.1 to 0.3 weight percent [wt\%]) and do not vary systematically with depth (Davis, Mottl, Fisher, et al., 1992). Only four samples of pyrite in sediment from Hole $855 \mathrm{C}$ were analyzed for sulfur isotope values (Table 1). The $\delta^{34} \mathrm{~S}$ values range from $-40.7 \%$ to $-5.4 \%$ and there is a poorly defined trend to higher values at depth (Fig. 1).

\section{Site 856}

\section{Hole 856A}

Hole $856 \mathrm{~A}$ was drilled in the center of a circular hill of uplifted sediment (Bent Hill). Sediment becomes increasingly indurated and is hydrothermally altered at depth in this hole (Davis, Mottl, Fisher, et al., 1992).

An olivine diabase sill was cored in the bottom $3 \mathrm{~m}$ of the hole. Sulfur content is generally between 0.1 and $0.3 \mathrm{wt} \%$ in the top $20 \mathrm{~m}$ of the hole and becomes more variable and tends towards higher values at depth (Davis, Mottl, Fisher, et al., 1992; Table 1). Disseminated pyrite has $\delta^{34} \mathrm{~S}$ values between $-39.8 \%$ and $17.7 \%$ (Fig. 2 ). The data indicate a general trend to higher values at depth.

\section{Hole $856 B$}

Hole 856B was drilled on the south flank of Bent Hill. In addition to the turbiditic and hemipelagic sediment that fills Middle Valley, an olivine diabase sill approximately $5 \mathrm{~m}$ thick occurs at $62 \mathrm{mbsf}$. Hole $856 \mathrm{~B}$ ended after drilling approximately $1.5 \mathrm{~m}$ into an olivine diabase sill at $120 \mathrm{mbsf}$. The recovered sediment was generally indurated to hydrothermally altered, especially near the basaltic sills. The bottom $18 \mathrm{~m}$ above the basaltic sill is a zone of hydrothermal alteration and sulfide mineralization that becomes more prominent toward the contact. Pyrite is the dominant sulfide mineral through most of the sediment section, but pyrrhotite, sphalerite, and chalcopyrite occur in the mineralized zone adjacent to the sill. Disseminations and veins of anhydrite and barite occur in this interval.

Pyrite is visibly more abundant in Hole $856 \mathrm{~B}$ than in Hole $856 \mathrm{~A}$. Sulfur content of the sediment is typically between 1 and $1.5 \mathrm{wt} \%$ (Davis, Mottl, Fisher, et al., 1992). An interval between 18 and 24 mbsf has much higher sulfur content due to the presence of turbidites and debris flows derived from massive sulfide. The $\delta^{34} \mathrm{~S}$ values of disseminated pyrite in the sediment range from $-22.8 \%$ to $31.3 \%$ (Table 1). Sulfur isotope values tend to become higher downhole at least to the depth of the first olivine diabase sill (Fig. 3). Below this depth sulfur isotope values of pyrite are fairly constant but range from $6.0 \%$ to $13.1 \%$. Sulfur isotope values for the interval containing clas- 
Table 1. Sulfur content and sulfur isotope values of Middle Valley samples.

\begin{tabular}{|c|c|c|c|c|c|c|c|c|c|}
\hline \multirow{2}{*}{$\begin{array}{l}\text { Core, section, } \\
\text { interval }(\mathrm{cm})\end{array}$} & \multirow{2}{*}{$\begin{array}{l}\text { Depth } \\
\text { (mbsf) }\end{array}$} & & & & & $\mathrm{s}$ & & & \\
\hline & & Lithology & (\%) & Py & Po & SI & An & $\mathrm{Ba}$ & Notes \\
\hline 139-855C. & & & & & & & & & \\
\hline $2 \mathrm{R}-4,139-143$ & 14.59 & Silt & 0.75 & -26.6 & & & & & \\
\hline $4 R-4,91-93$ & 32.51 & Clay & 1.84 & -40.7 & & & & & \\
\hline $10 \mathrm{R}-4,62-64$ & 88.52 & Sand & 1.71 & -5.4 & & & & & \\
\hline $11 R-1,101-106$ & 95.61 & Sand & 0.70 & -14.2 & & & & & \\
\hline $139-856 \mathrm{~A}-$ & & & & & & & & & \\
\hline $2 \mathrm{H}-3,76-78$ & 6.46 & Clay & 0.95 & -39.8 & & & & & \\
\hline $3 \mathrm{H}-3,30-32$ & 15.50 & Silt & 4.82 & -22.6 & & & & & \\
\hline $3 \mathrm{H}-3,142-146$ & 16.62 & Sand & 0.32 & -18.8 & & & & & \\
\hline $4 \mathrm{H}-1,47-49$ & 22.17 & Clay & 1.17 & -20.3 & & & & & \\
\hline $4 \mathrm{H}-1,95-97$ & 22.65 & Clay & 0.16 & & & & & & \\
\hline $4 \mathrm{H}-2,147-149$ & 24.67 & Clay & 0.11 & -4.7 & & & & & \\
\hline $4 \mathrm{H}-3,32-34$ & 25.02 & Clay & 0.79 & -24.8 & & & & & \\
\hline $4 \mathrm{H}-4,53-55$ & 26.73 & Clay & 0.04 & & & & & & \\
\hline $4 \mathrm{H}-6,80-82$ & 30.00 & Sand & 0.09 & -8.9 & & & & & \\
\hline $5 \mathrm{H}-7,103-105$ & 40.11 & Sand & 0.43 & -4.9 & & & & & \\
\hline $5 \mathrm{H}-8,13-15$ & 40.71 & Silt & 0.15 & -21.0 & & & & & \\
\hline $5 \mathrm{H}-8.86-88$ & 41.44 & Clay & 4.95 & 2.4 & & & & & \\
\hline $6 \mathrm{H}-3,65-67$ & 44.35 & Sand & 0.91 & 3.8 & & & & & \\
\hline $6 \mathrm{H}-4,64-66$ & 45.84 & Silt & 0.27 & 7.4 & & & & & \\
\hline $6 \mathrm{H}-6,1-3$ & 48.21 & Silt & 0.28 & & & & & & \\
\hline $6 \mathrm{H}-6,140-142$ & 49.60 & Silt & 0.12 & -18.1 & & & & & \\
\hline $7 \mathrm{H}-1,127-129$ & 51.47 & Silt & 1.55 & 6.8 & & & & & \\
\hline $7 \mathrm{H}-5,3-5$ & 56.23 & Clay & 0.18 & -22.4 & & & & & \\
\hline $8 \mathrm{H}-2,131-133$ & 62.51 & Silt & 0.31 & 7.1 & & & & & \\
\hline $8 \mathrm{H}-\mathrm{CC}, 28-30$ & 69.69 & Silt & 2.59 & -7.8 & & & & & \\
\hline $10 \mathrm{X}-1,131-133$ & 80.01 & Clay & 0.07 & & & & & & \\
\hline $11 X-1,32-34$ & 86.52 & Silt & 1.57 & -11.5 & & & & & \\
\hline $12 X-1,139-141$ & 97.09 & Silt & 0.69 & 17.7 & & & & & \\
\hline $13 X-1,111-113$ & 106.41 & Silt & 0.45 & 16.2 & & & & & \\
\hline $13 \times-3,68-70$ & 108.98 & Silt & 0.15 & 10.5 & & & & & \\
\hline 139-856B- & & & & & & & & & \\
\hline $2 \mathrm{H}-2,53-56$ & 3.83 & Silt & 0.65 & -22.8 & & & & & \\
\hline $2 \mathrm{H}-2,53-56$ & 3.83 & Silt & & -17.3 & & & & & \\
\hline $2 \mathrm{H}-3,25-29$ & 5.05 & Sand & 0.15 & -10.4 & & & & & \\
\hline $2 \mathrm{H}-5,8-12$ & 7.88 & Clay & 1.34 & -5.6 & & & & & \\
\hline $3 \mathrm{H}-2.87-89$ & 13.67 & Silt & 0.35 & -2.2 & & & & & \\
\hline $3 \mathrm{H}-5,137-139$ & 18.67 & CS & & 2.1 & & & & & \\
\hline $3 \mathrm{H}-6,5 \mathrm{I}-53$ & 19.31 & $\mathrm{CS}$ & & 3.0 & & & & & \\
\hline $3 \mathrm{H}-6,51-53$ & 19.31 & CS & & 3.1 & & & & & \\
\hline $3 \mathrm{H}-6,105-107$ & 19.85 & $\mathrm{CS}$ & & 4.7 & & & & & \\
\hline $3 \mathrm{H}-6,105-107$ & 19.85 & CS & & 4.4 & & & & & \\
\hline $4 \mathrm{H}-2,25-27$ & 22.55 & $\mathrm{CS}$ & & -1.1 & & & & & Total sulfide \\
\hline $4 \mathrm{H}-2,25-27$ & 22.55 & $\mathrm{CS}$ & & -1.2 & & & & & Fresh Py \\
\hline $4 \mathrm{H}-2,25-27$ & 22.55 & $\mathrm{CS}$ & & 2.3 & & & & & Weathered Py \\
\hline $4 \mathrm{H}-5,77-79$ & 27.57 & Silt & 9.82 & -8.1 & & & & & \\
\hline $5 \mathrm{H}-5,8-11$ & 36.38 & Silt & 1.05 & 6.0 & & & & & \\
\hline $6 \mathrm{H}-2,82-85$ & 42.12 & Clay & 0.58 & -7.0 & & & & & \\
\hline $6 \mathrm{H}-2,134-136$ & 42.64 & Clay & 0.22 & 3.7 & & & & & \\
\hline $6 \mathrm{H}-4,117-119$ & 45.47 & Clay & 0.60 & 8.0 & & & & & \\
\hline $7 \mathrm{H}-1,25-28$ & 49.55 & Silt & 0.32 & 3.2 & & & & & \\
\hline $7 \mathrm{H}-3,89-92$ & 53.19 & Silt & & 8.8 & & & & & Total sulfide \\
\hline $7 \mathrm{H}-3,89-92$ & 53.19 & Silt & & 9.5 & & & & & Py nodule \\
\hline $7 \mathrm{H}-3,89-92$ & 53.19 & Silt & & 10.4 & & & & & Py nodule \\
\hline $7 \mathrm{H}-3,89-92$ & 53.19 & Silt & & & & & 10.1 & & \\
\hline $7 \mathrm{H}-3,89-92$ & 53.19 & Silt & & & & & 4.2 & & \\
\hline $7 \mathrm{H}-4,67-70$ & 54.47 & Silt & & -0.8 & & & & & Total sulfide \\
\hline $7 \mathrm{H}-4,67-70$ & 54.47 & Silt & 0.94 & -16.8 & & & & & Py filled burrow \\
\hline $7 \mathrm{H}-6,44-47$ & 57.24 & Clay & 6.22 & 31.3 & & & & & \\
\hline $8 \mathrm{H}-4,42-44$ & 63.60 & Clay & 1.28 & 7.9 & & & & & \\
\hline $9 X-1,20-22$ & 63.86 & Silt & 1.32 & 4.3 & & & & & \\
\hline $11 X-5,42-45$ & 79.72 & Silt & 1.38 & 12.7 & & & & & \\
\hline $12 X-5,71-73$ & 88.31 & Silt & 5.01 & 7.7 & & & & & \\
\hline $12 \times-5,107-109$ & 88.67 & Clay & 0.38 & 11.4 & & & & & \\
\hline $12 \times-5,117-119$ & 88.77 & Clay & 0.39 & 10.1 & & & & & \\
\hline $12 X-5,117-119$ & 88.77 & Clay & & 8.8 & & & & & \\
\hline $13 \mathrm{X}-1,112-114$ & 92.22 & Clay & 3.17 & 13.1 & & & & & \\
\hline $13 \mathrm{X}-2,79-83$ & 93.39 & Clay & 0.25 & 10.8 & & & & & \\
\hline $14 X-4,24-31$ & 105.54 & Clay & & 9.5 & & & & & Total sulfide \\
\hline $14 X-4,24-31$ & 105.54 & Clay & & 7.2 & & 10.2 & & & Co-existing Py-SI \\
\hline $14 X-4,50-55$ & 105.80 & Clay & & 10.3 & & & & & \\
\hline 14X-CC, 34-38 & 106.51 & Sand & & 7.2 & & & & & \\
\hline $15 X-1,27-29$ & 110.67 & Clay & & 6.6 & & & & & \\
\hline $15 X-1,65-67$ & 111.05 & Clay & & 6.2 & & & & & \\
\hline $15 X-1,137-139$ & 111.77 & Clay & & 7.1 & & & & & \\
\hline $15 X-3,42-44$ & 113.82 & Clay & & 7.3 & & & & & \\
\hline $15 X-3,108-110$ & 114.48 & Clay & & 6.7 & & & & 22.7 & \\
\hline $15 \times-4,107-109$ & 115.97 & Clay & & & & & & 21.8 & \\
\hline $15 \times-4,128-130$ & 116.18 & Clay & & 6.5 & & & & 23.9 & \\
\hline $15 X-5,34-36$ & 116.74 & Clay & & 7.3 & & 6.9 & 21.6 & & \\
\hline $15 X-5,119-121$ & 117.59 & Silt & & & & & 23.2 & & \\
\hline $15 \mathrm{X}-\mathrm{CC}, 12-14$ & 118.14 & Clay & & 6.0 & & & & 23.8 & Py-Ba vein \\
\hline $16 X-1,17-19$ & 120.27 & Clay & & 6.0 & & 6.0 & & 22.2 & Py-Ba vein \\
\hline
\end{tabular}


Table 1 (continued).

\begin{tabular}{|c|c|c|c|c|c|c|c|c|c|}
\hline \multirow{2}{*}{$\begin{array}{l}\text { Core, section, } \\
\text { interval }(\mathrm{cm})\end{array}$} & \multirow{2}{*}{$\begin{array}{l}\text { Depth } \\
\text { (mbsf) }\end{array}$} & \multirow[b]{2}{*}{ Lithology } & & & & & & & \\
\hline & & & $(\%)$ & Py & Po & SI & An & $\mathrm{Ba}$ & Notes \\
\hline 139-856D- & & & & & & & & & \\
\hline $1 \mathrm{H}-1,43-44$ & 0.43 & MS & & 4.6 & & & & & \\
\hline $1 \mathrm{H}-1,43-44$ & 0.43 & MS & & 5.3 & & & & & \\
\hline $1 \mathrm{H}-3,140-150$ & 4.40 & MS & & 6.7 & & & & & \\
\hline IH $-3,140-150$ & 4.40 & MS & & 4.7 & & & & & \\
\hline IH $-4,34-35$ & 4.84 & MS & & 4.5 & & & & & \\
\hline $1 \mathrm{H}-4,79-82$ & 5.29 & MS & & 4.7 & & & & & \\
\hline $\begin{array}{l}\text { IH }-5,14-15 \\
\text { IH-5, 14-15 }\end{array}$ & $\begin{array}{l}6.14 \\
6.14\end{array}$ & $\begin{array}{l}\text { MS } \\
\text { MS }\end{array}$ & & $\begin{array}{l}4.5 \\
4.6\end{array}$ & & & & & \\
\hline $\begin{array}{l}1 \mathrm{H}-5,14-15 \\
1 \mathrm{H}-7,78-80\end{array}$ & 8.21 & MS & & 5.0 & & & & & \\
\hline IH- $7,78-80$ & 8.21 & MS & & 5.8 & & & & & \\
\hline $1 \mathrm{H}-7,78-80$ & 8.21 & MS & & 12.3 & & 7.5 & & & \\
\hline 139-856G- & & & & & & & & & \\
\hline $3 \mathrm{R}-1,7-8$ & 17.67 & MS & & 5.2 & & & & & \\
\hline $3 \mathrm{R}-1,7-8$ & 17.67 & MS & & 5.7 & & & & & \\
\hline $4 \mathrm{R}-1,131-133$ & 28.31 & MS & & 8.1 & & & & & \\
\hline $5 R-1,44-46$ & 37.14 & MS & & 6.3 & & & & & \\
\hline 6R-1, 36-38 & 46.66 & MS & & 7.5 & & 3.1 & & & Sl vein \\
\hline $\begin{array}{l}6 \mathrm{R}-1,36-38 \\
6 \mathrm{R}-2,140-142\end{array}$ & $\begin{array}{l}46.66 \\
49.20\end{array}$ & $\begin{array}{l}\text { MS } \\
\text { MS }\end{array}$ & & 8.3 & 8.3 & & & & \\
\hline $6 \mathrm{R}-2,140-142$ & 49.20 & MS & & 6.2 & & & & & \\
\hline $6 \mathrm{R}-3,24-26$ & 49.54 & MS & & 8.3 & & & & & \\
\hline $6 \mathrm{R}-3,79-81$ & 50.09 & MS & & 8.9 & & & & & \\
\hline $6 \mathrm{R}-3,79-81$ & 50.09 & MS & & 12.4 & & & & & \\
\hline $7 \mathrm{R}-2,27-29$ & 57.47 & MS & & 9.7 & & & & & \\
\hline $7 \mathrm{R} \cdot 2,27-29$ & 57.47 & MS & & 4.9 & & & & & \\
\hline $7 \mathrm{R}-3,98-100$ & 59.67 & MS & & 8.5 & & & & & \\
\hline $7 \mathrm{R}-3,98-100$ & $\begin{array}{l}59.67 \\
6014\end{array}$ & $\begin{array}{l}\text { MS } \\
\text { MS }\end{array}$ & & $\begin{array}{l}7.7 \\
80\end{array}$ & & 5.5 & & & \\
\hline 139-856 & 60.14 & Ms & & 0.0 & & & & & \\
\hline $\begin{array}{l}139-856 \mathrm{H}- \\
\text { IR-CC, 7-9 }\end{array}$ & 4.00 & MS & & 6.0 & & & & & \\
\hline $\begin{array}{l}\text { IR-CC, } 7-9 \\
\text { IR-CC, } 7-9\end{array}$ & 4.00 & MS & & 5.7 & & & & & \\
\hline $3 \mathrm{R}-1,78-80$ & 22.88 & MS & & 4.4 & & & & & \\
\hline $3 \mathrm{R}-1,78-80$ & 22.88 & MS & & 3.8 & & & & & \\
\hline $3 R-2,45-47$ & 23.71 & MS & & 5.7 & & & & & \\
\hline $3 R-3,24-26$ & 24.95 & MS & & 7.8 & & 8.3 & & & \\
\hline $4 \mathrm{R}-1,48-50$ & 27.08 & MS & & 6.7 & & & & & \\
\hline $4 \mathrm{R}-1,48-50$ & 27.08 & MS & & 5.8 & & & & & \\
\hline $4 \mathrm{R}-1,142-144$ & 28.02 & MS & & 5.9 & 7.6 & & & & \\
\hline $4 \mathrm{R}-1,142-144$ & 28.02 & MS & & 7.9 & & & & & Coarse Py \\
\hline $4 \mathrm{R}-1,142-144$ & 28.02 & MS & & & & 8.2 & & & Course SI \\
\hline $\begin{array}{l}4 \mathrm{R}-2,12-14 \\
4 \mathrm{R}-2,50-52\end{array}$ & $\begin{array}{l}28.22 \\
28.60\end{array}$ & $\begin{array}{l}\text { MS } \\
\text { MS }\end{array}$ & & $\begin{array}{l}7.5 \\
7.1\end{array}$ & & & & & Po clast \\
\hline $\begin{array}{l}4 R-2,50-52 \\
4 R-2,50-52\end{array}$ & $\begin{array}{l}28.60 \\
28.60\end{array}$ & $\begin{array}{l}\text { MS } \\
\text { MS }\end{array}$ & & 8.2 & 7.3 & & & & \\
\hline $5 R-1,27-28$ & 32.67 & MS & & 8.1 & 7.8 & & & & \\
\hline $6 \mathrm{R}-1,12-14$ & 37.52 & MS & & 6.4 & & & & & \\
\hline $6 R-1,12-14$ & 37.52 & MS & & 6.3 & & & & & \\
\hline $8 \mathrm{R}-1,30-32$ & 48.30 & MS & & 5.3 & & & & & \\
\hline $8 \mathrm{R}-1,30-32$ & 48.30 & MS & & 5.0 & & & & & \\
\hline $8 \mathrm{R}-1,115-117$ & 49.15 & MS & & 6.7 & & & & 20.9 & \\
\hline 9R-1, 7-9 & 52.57 & MS & & 7.0 & & & & & \\
\hline $10 \mathrm{R}-1,50-51$ & 57.50 & MS & & 2.6 & & & & & \\
\hline 11R-1, 107-109 & 62.37 & MS & & 1.9 & & & & & \\
\hline $13 \mathrm{R}-1,2-4$ & 70.92 & MS & & 6.7 & & & & & \\
\hline $14 \mathrm{R}-1,132-134$ & 77.02 & MS & & & 5.0 & & & & \\
\hline $14 \mathrm{R}-1,132-134$ & 77.02 & MS & & 6.9 & 6.4 & & & & \\
\hline $14 \mathrm{R}-1,132-134$ & 77.02 & MS & & 5.6 & & & & & Py porphyroblast \\
\hline $14 \mathrm{R}-1,132-134$ & 77.02 & MS & & -8.0 & & & & & Late vug filling Py \\
\hline $15 \mathrm{R}-1,127-129$ & 81.77 & MS & & 7.5 & & & & & \\
\hline $16 \mathrm{R}-1,4-6$ & 85.24 & MS & & 4.9 & 5.0 & & & & \\
\hline $16 \mathrm{R}-1,4-6$ & 85.24 & MS & & -1.9 & & & & & Late vug filling Py \\
\hline $16 \mathrm{R}-1,31-32$ & 85.51 & MS & & 5.0 & & & & & \\
\hline $17 \mathrm{R}-1,33-34$ & 90.43 & MS & & 6.7 & 6.7 & & & & \\
\hline $17 \mathrm{R}-1,33-34$ & 90.43 & MS & & 2.5 & & & & & Chalcopyrite \\
\hline $139-857 \mathrm{~A}-$ & & & & & & & & & \\
\hline $4 \mathrm{H}-3,119-121$ & 26.09 & Clay & 1.85 & -30.3 & & & & & \\
\hline $5 \mathrm{H}-4,81-83$ & 36.71 & Clay & 4.63 & -21.7 & & & & & \\
\hline $6 \mathrm{H}-2,102-106$ & 43.42 & Silt & 0.20 & -8.0 & & & & & \\
\hline $8 \mathrm{H}-3,117-119$ & 64.07 & Silt & 0.30 & 0.6 & & & & & \\
\hline $8 \mathrm{H}-3,117-119$ & 64.07 & Silt & & 3.1 & & & & & Py nodule \\
\hline $10 \mathrm{H}-2,123-125$ & 81.63 & Silt & 1.54 & 9.0 & & & & & \\
\hline $13 X-1,117-119$ & 102.67 & Silt & 0.83 & 11.9 & & & & & \\
\hline 139-857C- & & & & & & & & & \\
\hline $2 \mathrm{R}-1,28-31$ & 56.78 & Silt & 1.33 & -12.0 & & & & & \\
\hline $2 \mathrm{R}-1,28-31$ & 56.78 & Silt & & & & & -11.2 & & \\
\hline $2 \mathrm{R}-1,28-31$ & 56.78 & Silt & & & & & -6.6 & & \\
\hline $3 R-2,106-108$ & 69.06 & Silt & 0.14 & -16.7 & & & & & \\
\hline $13 \mathrm{R}-1,62-64$ & 153.72 & Sand & 3.41 & 2.0 & & & & & \\
\hline $14 \mathrm{R}-1,47-51$ & 163.27 & Clay & 0.41 & 6.6 & & & & & \\
\hline $15 \mathrm{R}-1,1-3$ & 172.51 & Sand & 0.09 & 17.2 & & & & & \\
\hline $15 R-1,61-63$ & 173.11 & Silt & 0.26 & 18.0 & & & & & \\
\hline $17 \mathrm{R}-2,84-88$ & 194.24 & Silt & 0.09 & -8.4 & & & & & \\
\hline $22 \mathrm{R}-1,144-147$ & 241.74 & Clay & 0.14 & -0.5 & & & & & \\
\hline $30 \mathrm{R}-2,147-150$ & 316.07 & Silt & 0.03 & 10.5 & & & & & \\
\hline $31 R-3,14-16$ & 325.84 & Silt & 0.10 & -2.2 & & & & & \\
\hline
\end{tabular}


Table 1 (continued).

\begin{tabular}{|c|c|c|c|c|c|c|c|c|c|}
\hline \multirow{2}{*}{$\begin{array}{l}\text { Core, section, } \\
\text { interval }(\mathrm{cm})\end{array}$} & & & & & & & & & \\
\hline & (mbsf) & Lithology & (\%) & $\mathrm{Py}$ & Po & SI & An & $\mathrm{Ba}$ & Notes \\
\hline $31 \mathrm{R}-\mathrm{CC}, 0-3$ & 326.02 & Clay & 0.49 & 1.5 & & & & & \\
\hline $33 \mathrm{R}-1,100-102$ & 333.40 & Silt & 0.21 & 6.4 & & & & & \\
\hline $33 R-1,123-125$ & 333.63 & Silt & 0.27 & 9.1 & & & & & \\
\hline $33 R-2,133-135$ & 335.23 & Clay & 0.29 & 6.8 & & & & & \\
\hline $34 \mathrm{R}-3,37-39$ & 339.87 & Silt & 0.26 & -1.6 & & & & & \\
\hline $34 R-3,67-70$ & 340.17 & Clay & 0.23 & -25.4 & & & & & \\
\hline $35 \mathrm{R}-1,66-68$ & 341.96 & Silt & 0.12 & -26.5 & & & & & \\
\hline $35 \mathrm{R}-2,44-46$ & 343.24 & Clay & & -12.0 & & & & & \\
\hline $37 \mathrm{R}-2,121-123$ & 353.51 & Clay & & -11.3 & & & & & \\
\hline $38 \mathrm{R}-1,4-6$ & 355.84 & Clay & & -9.6 & & & & & Burrow fill py \\
\hline $39 \mathrm{R}-1,119-12 \mathrm{I}$ & 361.59 & Clay & & 38.8 & & & & & \\
\hline $41 \mathrm{R}-1,144-146$ & 376.34 & Clay & & -1.2 & & & & & \\
\hline $42 R-2,29-31$ & 381.49 & Silt & & 15.0 & & & & & \\
\hline $43 R-3,0-4$ & 387.70 & Sand & & 54.3 & & & & & MS clasts in turbidite \\
\hline $49 R-1,111-114$ & 414.81 & Clay & & 29.1 & & & & & \\
\hline $50 \mathrm{R}-2,58-60$ & 420.38 & Clay & & 16.8 & & & & & Py vein \\
\hline $50 R-2,75-77$ & 420.55 & Clay & & 15.3 & & & & & Py nodule \\
\hline $51 R-2,18-20$ & 425.08 & Sand & & 13.6 & & & & & \\
\hline $56 \mathrm{R}-1,0-2$ & 446.50 & Clay & & 12.4 & & & & & Py vein \\
\hline $57 \mathrm{R}-1,11-12$ & 451.81 & Sand & & 14.3 & & & & & Coarse euhedral Py \\
\hline $58 \mathrm{R}-1,2-3$ & 461.52 & Clay & & 12.8 & & & & & Py vein \\
\hline $59 \mathrm{R}-1,3-4$ & 471.13 & Diabase & & 9.3 & & & & & $\mathrm{Py}-\mathrm{Chl}$ vein \\
\hline $59 \mathrm{R}-1,3-4$ & 471.13 & Diabase & & & & 7.6 & & & Si-Cp-Wai vein \\
\hline $59 \mathrm{R}-4,72-74$ & 476.24 & Diabase & & -0.2 & & & & & Chl-Py selvage of Epi vein \\
\hline $60 \mathrm{R}-1,126-128$ & 482.06 & Diabase & & 8.0 & & & & & \\
\hline $61 \mathrm{R}-1,41-43$ & 490.91 & Diabase & & 8.4 & & & & & \\
\hline $62 \mathrm{R}-1,120-130$ & 501.20 & Diabase & & 3.6 & & & & & \\
\hline $63 \mathrm{R}-1,29-31$ & 509.99 & Silt & & 13.6 & & & & & \\
\hline $64 \mathrm{R}-2,57-59$ & 521.47 & Diabase & & & & 7.5 & & & Wai-SI vein \\
\hline $65 R-1,2-4$ & 529.12 & Diabase & & 7.5 & & & & & \\
\hline $66 \mathrm{R}-1,2-3$ & 538.82 & Sand & & 15.5 & & & & & \\
\hline $68 \mathrm{R}-1,8-10$ & 558.08 & Diabase & & 5.2 & & & & & \\
\hline 139-857D- & & & & & & & & & \\
\hline IR-1, $42-44$ & 581.92 & Diabase & & & & 7.7 & & & SI-Cp Wai vein \\
\hline $3 R-1,62-63$ & 599.92 & Diabase & & 8.4 & & & & & Selvage of Epi-Qtz-Py-Cp vein \\
\hline $4 \mathrm{R}-1,133-136$ & 610.23 & Diabase & & 8.1 & & & & & \\
\hline $5 R-1,16-18$ & 618.76 & Sand & & 9.6 & 8.3 & & & & \\
\hline $6 \mathrm{R}-1,2-4$ & 627.92 & Silt & & & 8.7 & & & & Qtz Po vein \\
\hline $8 \mathrm{R}-1,31-33$ & 647.61 & Diabase & & 9.4 & & & & & $\begin{array}{l}\text { Py-Chl vein in quenched } \\
\text { margin }\end{array}$ \\
\hline $9 R-1,25-27$ & 657.15 & Diabase & & 9.3 & & & & & Py Wai? vein \\
\hline $12 \mathrm{R}-1,75-77$ & 686.65 & Diabase & & & 8.2 & & & & Po-Wai-Qtz vein \\
\hline $12 \mathrm{R}-1,121-124$ & 687.11 & Diabase & & 8.5 & & & & & Wai-Qtz-Py vein \\
\hline $15 \mathrm{R}-1,55-57$ & 715.35 & Diabase & & 10.2 & & & & & \\
\hline $16 \mathrm{R}-1,48-50$ & 724.98 & Silt & & 13.0 & & & & & \\
\hline $17 \mathrm{R}-1,96-98$ & 734.86 & Silt & & 14.6 & & & & & \\
\hline $18 \mathrm{R}-1,94-96$ & 744.54 & Diabase & & 7.2 & & & & & \\
\hline $22 \mathrm{R}-1,45-46$ & 782.25 & Diabase & & 11.1 & & & & & Py-Epi vein \\
\hline $24 \mathrm{R}-1,100-102$ & 802.20 & Clay & & 11.4 & & & & & Py vein \\
\hline $24 \mathrm{R}-2,45-47$ & 803.15 & Diabase & & 12.0 & & & & & \\
\hline $26 \mathrm{R}-1,39-41$ & 820.69 & Diabase & & 10.0 & & & & & \\
\hline $27 \mathrm{R}-1,22-24$ & 829.22 & Diabase & & 9.9 & & 8.6 & & & Qtz-Wai-Epi-Sl-Py vein \\
\hline $28 \mathrm{R}-1,50-52$ & 839.40 & Silt & & 9.0 & & & & & \\
\hline $29 \mathrm{R}-2,40-42$ & 850.30 & Diabase & & 11.2 & & & & & \\
\hline $31 \mathrm{R}-1,20-24$ & 868.70 & Sand & & 10.9 & & & & & \\
\hline $33 \mathrm{R}-1,92-93$ & 888.72 & Diabase & & 7.9 & & & & & Epi-Py vein \\
\hline $36 \mathrm{R}-1,129-131$ & 918.19 & Diabase & & 7.7 & & & & & \\
\hline $139-858 \mathrm{~A}-$ & & & & & & & & & \\
\hline $2 \mathrm{H}-7,34-38$ & 11.74 & Clay & 0.60 & 3.4 & & & & & \\
\hline $3 \mathrm{H}-1,87-91$ & 12.77 & Silt & 0.71 & -5.6 & & & & & \\
\hline $5 \mathrm{H}-5,65-68$ & 37.55 & Silt & 0.19 & 7.6 & & & & & \\
\hline $6 \mathrm{H}-3,144-146$ & 44.84 & Silt & 1.50 & -2.3 & & & & & \\
\hline $6 \mathrm{H}-\mathrm{CC}, 7-9$ & 44.97 & Clay & 1.73 & -5.3 & & & & & \\
\hline $7 \mathrm{H}-4,105-108$ & 55.46 & Silt & 0.79 & 16.0 & & & & & \\
\hline $9 X-2,136-138$ & 65.36 & Silt & 0.04 & 5.9 & & & & & \\
\hline $9 \times-3,10-12$ & 65.60 & Clay & 1.27 & 25.6 & & & 24.2 & & An-Gyp cement \\
\hline $9 X-3,10-12$ & 65.60 & Clay & & 9.8 & & & & & Coarse Py \\
\hline $9 \times-3,61-63$ & 66.11 & Clay & 0.22 & 11.2 & & & & & \\
\hline $9 X-5,96-98$ & 69.46 & Clay & 0.56 & -21.0 & & & & & \\
\hline $11 \mathrm{X}-\mathrm{CC}, 7-8$ & 73.54 & Clay & 1.20 & 1.3 & & & & & \\
\hline $12 X-C C, 5-6$ & 81.85 & Sand & & 22.3 & & & & & \\
\hline $14 \mathrm{X}-\mathrm{CC}, 3-5$ & 101.03 & Sand & & -9.7 & & & & & \\
\hline $17 X-C C, 2-5$ & 130.27 & Clay & & & & & 21.3 & & Dissm bladed An \\
\hline $18 X-1,6-8$ & 139.66 & Sand & & & & & 26.3 & & \\
\hline $18 \times-2,127-130$ & 142.37 & Silt & & 28.9 & & & 25.0 & & \\
\hline $18 \mathrm{X}-\mathrm{CC}, 6-8$ & 143.04 & Clay & & & & & 21.9 & & \\
\hline $19 X-1,43-45$ & 149.73 & Silt & & & & & 28.3 & & \\
\hline $20 X-3,135-138$ & 163.28 & Clay & & 25.0 & & & & & An-Gyp vein \\
\hline $20 \times-4,15-18$ & 163.59 & Clay & & 27.0 & & & & & \\
\hline $21 \times-1,67-70$ & 169.27 & Sand & & 14.3 & & & 26.4 & & \\
\hline $21 \times-2,28-31$ & 170.38 & Clay & & & & & 25.0 & & An-Gyp vein \\
\hline $21 \times-2,71-74$ & 170.81 & Silt & & 1.9 & & & 22.4 & & \\
\hline $22 X-1,10-11$ & 178.30 & Clay & & 12.0 & & & 27.4 & & Dissm bladed An \\
\hline $23 X-1,71-73$ & 188.61 & Clay & & 14.2 & & & & & \\
\hline $24 X-1,61-63$ & 198.21 & Silt & & -32.4 & & & & & \\
\hline $26 \mathrm{X}-\mathrm{CC}, 6-8$ & 216.96 & Clay & 0.37 & 7.7 & & & 25.8 & & \\
\hline
\end{tabular}


Table 1 (continued).

\begin{tabular}{|c|c|c|c|c|c|c|c|c|c|}
\hline \multirow[b]{2}{*}{$\begin{array}{l}\text { Core, section, } \\
\text { interval }(\mathrm{cm})\end{array}$} & \multirow[b]{2}{*}{$\begin{array}{l}\text { Depth } \\
\text { (mbsf) }\end{array}$} & \multirow[b]{2}{*}{ Lithology } & \multirow[b]{2}{*}{$\underset{(\%)}{S}$} & \multicolumn{5}{|c|}{$\delta^{34} \mathrm{~S}$} & \multirow[b]{2}{*}{ Notes } \\
\hline & & & & Py & Po & $\mathrm{SI}$ & An & $\mathrm{Ba}$ & \\
\hline $29 \mathrm{X}-1,7-11$ & 245.97 & Sand & & 8.3 & & & & & \\
\hline $30 X-1,41-44$ & 256.01 & Clay & & 8.2 & & & & & \\
\hline $31 X-1,26-28$ & 265.56 & Clay & & 9.3 & & & & & Coarse Py cubes \\
\hline $38 \mathrm{X}-\mathrm{CC}, 10-12$ & 322.80 & Sand & & 9.2 & & & & & \\
\hline \multicolumn{10}{|l|}{ 139-858B- } \\
\hline $1 \mathrm{H}-2,27-29$ & 1.77 & Clay & 0.49 & -0.5 & & & & & \\
\hline $1 \mathrm{H}-5,3-5$ & 6.03 & Silt & 0.54 & -13.7 & & & & & \\
\hline $1 \mathrm{H}-\mathrm{CC}, 20-22$ & 7.10 & Clay & & -15.3 & & & & 44.6 & \\
\hline $2 \mathrm{H}-3,27-29$ & 10.47 & Clay & & 4.5 & & & & & Semi-MS \\
\hline $2 \mathrm{H}-3,119-121$ & 11.39 & Clay & & 4.1 & & & 26.3 & & Semi-MS \\
\hline $2 \mathrm{H}-4,7-9$ & 11.77 & Clay & & 1.7 & & & 23.7 & & Semi-MS \\
\hline \multicolumn{10}{|l|}{ 139-858C- } \\
\hline $3 \mathrm{H}-2,101-104$ & 15.51 & Clay & & 8.5 & & & 5.9 & & \\
\hline $3 \mathrm{H}-3,133-135$ & 17.33 & Clay & & 15.2 & & & 14.7 & & \\
\hline $5 \mathrm{H}-1,139-141$ & 24.98 & Silt & & -2.4 & & & & & \\
\hline $5 \mathrm{H}-5,27-29$ & 29.77 & Silt & & -2.8 & & & 3.0 & & Gyp \\
\hline $6 \mathrm{H}-1,143-145$ & 34.43 & Silt & & 3.6 & & & 22.6 & & An vein \\
\hline $\begin{array}{l}6 \mathrm{H}-3,82-83 \\
7 \mathrm{H}-1,32-34\end{array}$ & $\begin{array}{l}36.82 \\
41.82\end{array}$ & $\begin{array}{l}\text { Clay } \\
\text { Clay }\end{array}$ & & $\begin{array}{l}1.8 \\
4.3\end{array}$ & & & $\begin{array}{l}22.3 \\
22.5\end{array}$ & & An vein \\
\hline $7 \mathrm{H}-2,138-141$ & 44.38 & Clay & & -0.4 & & & & & \\
\hline $8 \mathrm{H}-1,58-59$ & 47.08 & Silt & & -3.4 & & & 22.7 & & Coarse Py, An nodule \\
\hline 10X-CC, 6-9 & 49.56 & Silt & & 1.5 & & & & & \\
\hline $11 \mathrm{X}-\mathrm{CC}, 30-32$ & 55.78 & Silt & & 9.0 & & & & & \\
\hline $12 \mathrm{X}-1,18-20$ & 64.18 & Silt & & 4.8 & & & & & \\
\hline $13 \mathrm{X}-1,2-5$ & 73.72 & Silt & & 8.3 & & & & & \\
\hline $13 X-1,41-46$ & 74.14 & Clay & & 8.0 & & & 23.8 & & \\
\hline $14 \mathrm{X}-\mathrm{CC}, 28-30$ & 84.46 & Silt & & -9.2 & & & 12.3 & & \\
\hline \multicolumn{10}{|l|}{ 139-858D- } \\
\hline $2 \mathrm{H}-4,10-13$ & 13.90 & Clay & & 15.1 & & & & & \\
\hline $4 \mathrm{H}-3,138-140$ & 22.92 & Clay & & -18.7 & & & 23.5 & & \\
\hline $4 \mathrm{H}-4,56-58$ & 23.60 & Silt & & 4.9 & & & 23.2 & & \\
\hline $6 \mathrm{X}-1,61-64$ & 29.41 & Clay & & 6.6 & & & & & \\
\hline \multicolumn{10}{|l|}{$139-858 \mathrm{~F}-$} \\
\hline 11R-CC, $12-14$ & 114.09 & Sand & & & & & 23.4 & & \\
\hline 13R-CC, $0-2$ & 132.90 & Clay & & 14.7 & & & & & \\
\hline $\begin{array}{l}\text { 13R-CC, 15-17 } \\
14 \mathrm{R}-1,16-17\end{array}$ & $\begin{array}{l}133.05 \\
142.76\end{array}$ & $\begin{array}{l}\text { Sand } \\
\text { Silt }\end{array}$ & & 12.1 & & & & & \\
\hline 15R-CC, $1-2$ & 152.21 & Silt & & 11.6 & & & 22.2 & & \\
\hline $19 \mathrm{R}-1,26-27$ & 191.16 & Silt & & 11.5 & & & & & \\
\hline $19 \mathrm{R}-1,37-38$ & 191.27 & Sand & & 11.1 & & & & & \\
\hline $21 \mathrm{R}-1,14-16$ & 210.44 & Clay & & 11.0 & & & 11.4 & & \\
\hline $21 \mathrm{R}-1,14-16$ & 210.44 & Clay & & 12.7 & & & & & \\
\hline 22R-CC, $12-13$ & 220.12 & Silt & & 9.3 & & & & & \\
\hline 23R-CC, 6-7 & 229.66 & Clay & & 6.3 & & & & & \\
\hline 24R-CC, 8-10 & 239.38 & Clay & & -12.6 & & & & & \\
\hline $\begin{array}{l}24 \mathrm{R}-\mathrm{CC}, 8-10 \\
25 \mathrm{R}-1,36-37\end{array}$ & $\begin{array}{l}239.38 \\
249.26\end{array}$ & $\begin{array}{l}\text { Clay } \\
\text { Silt }\end{array}$ & & -13.2 & & & & & \\
\hline $\begin{array}{l}25 \mathrm{R}-1,36-37 \\
25 \mathrm{R}-1,36-37\end{array}$ & $\begin{array}{l}249.20 \\
249.26\end{array}$ & $\begin{array}{l}\text { Sult } \\
\text { Silt }\end{array}$ & & $\begin{array}{l}11.1 \\
11.4\end{array}$ & & & & & \\
\hline $25 \mathrm{R}-1,75-76$ & 249.65 & Silt & & 12.5 & & & & & \\
\hline $25 \mathrm{R}-1,107-108$ & 249.97 & Basalt & & 13.3 & & & & & Qtz-Wai-Py vein \\
\hline $25 R-1,111-112$ & 250.01 & Basalt & & 13.8 & & & & & Qtz-Wai-Py vein \\
\hline $28 \mathrm{R}-1,19-20$ & 277.69 & Basalt & & 4.0 & & & & & \\
\hline \multicolumn{10}{|l|}{ 139-858G- } \\
\hline $2 \mathrm{R}-1,65-67$ & 287.15 & Basalt & & 5.8 & & & & & Py-Chi vein \\
\hline $4 \mathrm{H}-1,23-25$ & 306.03 & Basalt & & & & & 22.5 & & An vein \\
\hline $\begin{array}{l}5 \mathrm{R}-1,1-3 \\
6 \mathrm{R}-1,29-31\end{array}$ & $\begin{array}{l}315.41 \\
325.39\end{array}$ & $\begin{array}{l}\text { Basalt } \\
\text { Basalt }\end{array}$ & & 8.1 & 10.3 & & & & \\
\hline $10 \mathrm{R}-1,43-45$ & 365.33 & Basalt & & $\begin{array}{l}0.1 \\
9.7\end{array}$ & & & & & Py-cio vetio \\
\hline $13 \mathrm{R}-1,45-47$ & 394.35 & Basalt & & 8.8 & & & & & \\
\hline $16 R-1,88-89$ & 423.78 & Basalt & & 7.3 & & & & & \\
\hline
\end{tabular}

Notes: $\mathrm{MS}=$ massive sulfide, $\mathrm{CS}=$ clastic sulfide, $\mathrm{Py}=$ pyrite, $\mathrm{Po}=$ pyrnhotite, $\mathrm{SI}=$ sphalerite, $\mathrm{Cp}=$ chalcopyrite, $\mathrm{An}=$ anhydrite, $\mathrm{Gyp}=$ gypsum, $\mathrm{Ba}=$ barite, $\mathrm{Qtz}=$ quartz, $\mathrm{Chl}=$ chlorite, $\mathrm{Wai}=$ wairakite, $\mathrm{Epi}=$ epidote, $\mathrm{Dissm}=$ disseminated.

ticly deposited massive sulfide have a narrow range from $-1.2 \%$ to $4.7 \%$. Sphalerite was separated from three samples of the mineralized zone at the base of the hole. Sphalerite $\delta^{34} \mathrm{~S}$ values range from $6.0 \%$ to $10.2 \%$ and are not in isotopic equilibrium with coexisting pyrite. Disseminated anhydrite and barite from this interval are slightly enriched in ${ }^{34} \mathrm{~S}$ relative to seawater sulfate and range in value from $21.6 \%$ to $23.9 \%$; barite in veins gave values of $22.2 \%$ and $23.8 \%$. Two samples of disseminated sulfate (probably gypsum) in silt from 53.19 mbsf have values of $10.1 \%$ and $4.2 \%$, consistent with derivation of sulfate by local oxidation of pyrite from this interval (Table 1).

\section{Hole $856 \mathrm{D}$}

Hole $856 \mathrm{D}$ was piston cored into the weathered, rubbly top of a ridge of massive sulfide that occurs on the south flank of Bent Hill. Recovered material was disaggregated and partly weathered massive sulfide composed predominately of sand- to silt-sized pyrite grains and aggregates, with less abundant amorphous silica, sphalerite, pyrrhotite, and chalcopyrite. Pyrite from this core has $\delta^{34} S$ values of $4.6 \%$ to $5.7 \%$ except for one sample fragment from near the base of the core at 8.21 mbsf that yields values of $12.3 \%$ and $7.5 \%$ for coex- 


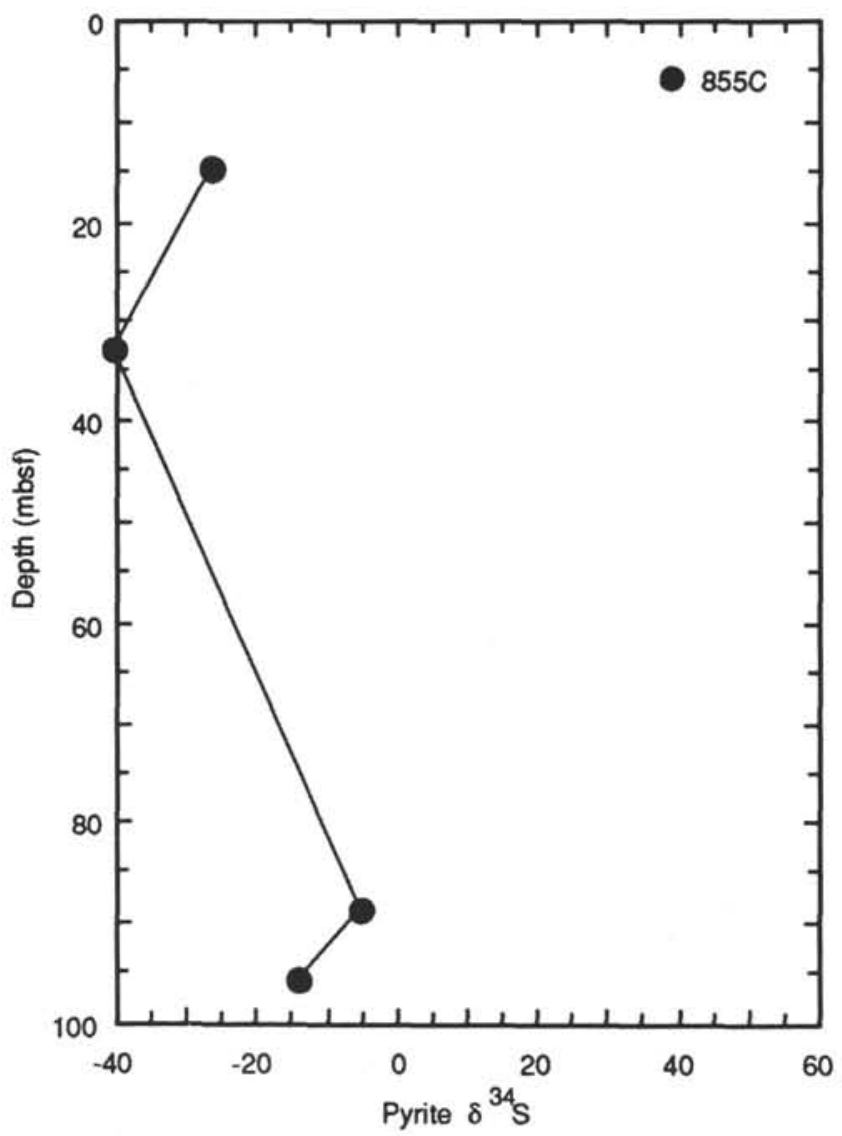

Figure 1. Sulfur isotope value of pyrite vs. depth for Hole 855C.

isting pyrite and sphalerite, respectively. These values are not consistent with isotopic equilibrium between the two sulfide minerals.

\section{Hole $856 G$}

Hole $856 \mathrm{G}$ was rotary cored near the top of the ridge of massive sulfide on the south flank of Bent Hill. This hole penetrated $65.4 \mathrm{~m}$ of massive sulfide before the hole was abandoned due to adverse drilling conditions. Massive sulfide recovered in this hole is described in Davis, Mottl, Fisher, et al. (1992). Pyrite from Hole 856G ranges in $\delta^{34} \mathrm{~S}$ value from $4.9 \%$ to $12.4 \%$, but most values are between $5 \%$ and $9 \%$ (Fig. 4). Coexisting pyrrhotite and pyrite from Sample 139$856 \mathrm{G}-6 \mathrm{R}-2,140-142 \mathrm{~cm}$ have identical values of $8.3 \%$, which is indicative of nonequilibrium but is consistent with pyrite inheriting pyrrhotite sulfur during replacement. Coexisting pyrite and sphalerite in Sample 139-856G-7R-4, 4-6 cm have values of $8.0 \%$ and $5.5 \%$, respectively, which are also incompatible with isotopic equilibrium at temperatures above $0^{\circ} \mathrm{C}$. A late-stage sphalerite vein in Sample 139$856 \mathrm{G}-6 \mathrm{R}-1,36-38 \mathrm{~cm}$ has a value of $3.1 \%$, which is $4.4 \%$ lighter than pyrite in the wall rock. The sulfur isotope values of massive sulfide in Hole $856 \mathrm{G}$ do not vary systematically between the different sulfide types defined in Davis, Mottl, Fisher, et al. (1992).

\section{Hole $856 \mathrm{H}$}

Hole $856 \mathrm{H}$ was rotary cored near the top of the ridge of massive sulfide on the south flank of Bent Hill adjacent to Hole $856 \mathrm{G}$. This hole penetrated $93.8 \mathrm{~m}$ of massive sulfide before the hole was abandoned due to adverse drilling conditions. Massive sulfide recovered in this hole is described in Davis, Mottl, Fisher, et al. (1992). Pyrite from Hole $856 \mathrm{H}$ ranges in $\delta^{34} \mathrm{~S}$ value from $-8.0 \%$ to $8.2 \%$, but most values are between $5 \%$ and $9 \%$, similar to samples from Hole $856 \mathrm{G}$ (Fig. 5). Two samples of paragenetically late, vug-filling pyrite have

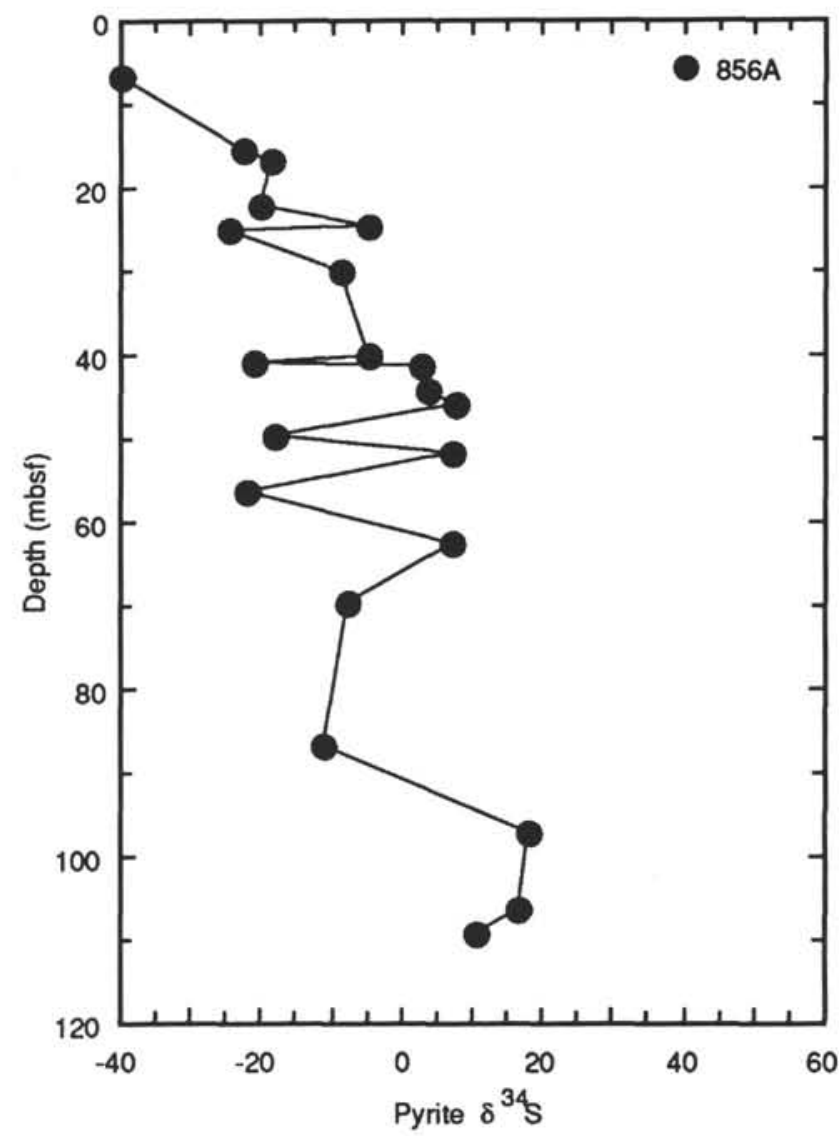

Figure 2. Sulfur isotope value of pyrite vs. depth for Hole 856A.

negative sulfur isotope values $(-1.9 \%$ and $-8.0 \%)$. Pyrrhotite values range from $5.0 \%$ to $7.8 \%$. With one exception, coexisting pyrite and pyrrhotite have $\delta^{34} \mathrm{~S}$ values that are within $0.5 \%$; in Sample 139 $856 \mathrm{H}-4 \mathrm{R}-1,142-144$ the pyrrhotite value is within $0.3 \%$ of coarsegrained pyrite "porphyroblasts" that replace pyrrhotite. However, none of the sample pairs appear to represent isotopic equilibrium. They are consistent with pyrite forming by replacement of pyrrhotite through a process that conserves sulfur, as is suggested by petrographic observations. Two microdrilled samples of sphalerite give values of $8.2 \%$ and $8.3 \%$, near the upper range of values for pyrite in this hole. One sample of chalcopyrite has a relatively low sulfur isotope value of $2.5 \%$. One barite sample gives a value of $20.9 \%$, identical to seawater sulfate. As in Hole $856 \mathrm{G}$, sulfur isotope values of massive sulfide in $856 \mathrm{H}$ do not vary systematically with sulfide type.

\section{Site 857}

Site 857 was drilled in an area of relatively high heat flow (700$800 \mathrm{~mW}$ ) about $1.6 \mathrm{~km}$ south of the large hydrothermal vent field at Site 858 . Samples for sulfur isotope analyses were collected from Holes $857 \mathrm{~A}, 857 \mathrm{C}$, and $857 \mathrm{D}$. Hole $857 \mathrm{~A}$ was drilled by advanced piston core-extended core barrel (APC-XCB) to $112 \mathrm{~m}$ depth. Holes 857C and 857D were drilled about $200 \mathrm{~m}$ east of Hole 857A and were rotary cored. At both Holes $857 \mathrm{C}$ and $857 \mathrm{D}$, interlayered diabase sills and metasedimentary rock were encountered at depths greater than $460 \mathrm{~m}$. Hole 857D was the deepest penetration in the Middle Valley area with a total depth of $936.2 \mathrm{~m}$.

\section{Hole 857 A}

Core recovered from Hole 857A consisted of interbedded turbiditic and hemipelagic sediment. Pore-water sulfate in Hole 857A de- 


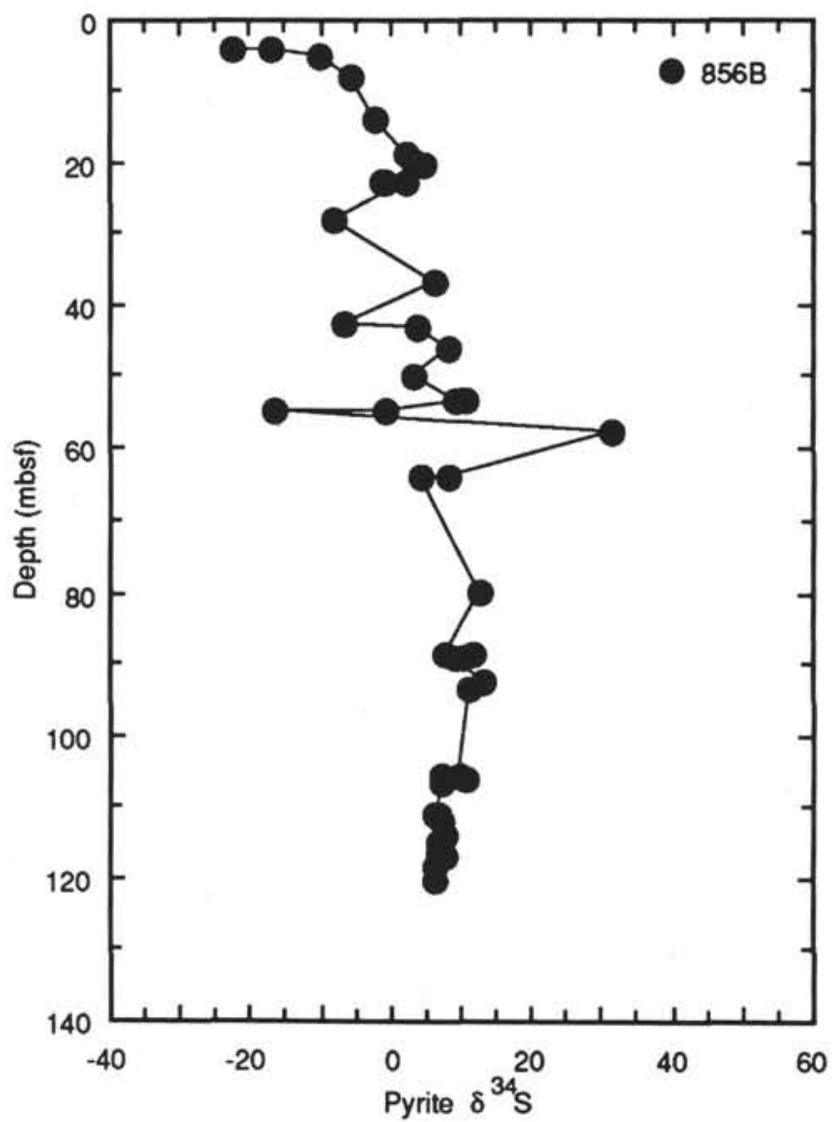

Figure 3. Sulfur isotope value of pyrite vs. depth for Hole 856B.

creases downhole to about two-thirds of bottom-water values due to bacterial reduction of sulfate (Davis, Mottl, Fisher, et al., 1992). Sulfur content of sediment is generally less than $0.5 \mathrm{wt} \%$ (Davis, Mottl, Fisher, et al., 1992). Sulfur isotope values of pyrite in sediment increase from $-30.3 \%$ at 26 mbsf to $11.9 \%$ at 103 mbsf (Fig. 6). A pyrite nodule at $64 \mathrm{~m}$ depth is $2.5 \%$ higher than disseminated pyrite in the sediment.

\section{Hole $857 C$}

Turbiditic and hemipelagic sediment in Hole $857 \mathrm{C}$ becomes increasingly lithified with depth. Basaltic sills are interbedded with metasedimentary rock below $471 \mathrm{~m}$. Pore-water sulfate values decrease downhole to a depth of about 200 mbsf, below which sulfate content is relatively constant at approximately $5 \mathrm{mmol} / \mathrm{kg}$ (Davis, Mottl, Fisher, et al., 1992). Sulfur content of the sediment is generally less than $0.5 \%$. Pyrite $\delta^{34} \mathrm{~S}$ values in Hole $857 \mathrm{C}$ have a wide range of values, from $-26.5 \%$ to $54.3 \%$ (Fig. 6). Pyrite $\delta^{34} \mathrm{~S}$ values in sediment are similar to those in Holes 856A, 856B, and 857A in that they generally increase with depth and show less variation with depth, approaching a value near $13 \%$. Pyrite burrow fills, veins, and nodules all have values similar to disseminated pyrite in nearby sediment. The extreme value of $54.3 \%$ is from the base of a coarse sand turbidite at 387.7 mbsf that contains clasts of massive sulfide composed of pyrite, pyrrhotite, and sphalerite (Davis, Mottl, Fisher, et al., 1992). These clasts of massive sulfide are the only evidence of a much earlier phase of hydrothermal activity in Middle Valley. This extremely heavy sulfur isotope value is uncommon in seafloor hydrothermal systems.

Two samples of sulfate from $56.78 \mathrm{mbsf}$ were analyzed. A leak in the vacuum extraction line occurred during conversion of the sample, yielding a value of $-6.6 \%$. This leak was partially fixed before the sample conversion was repeated, yielding a value of $-11.2 \%$. This

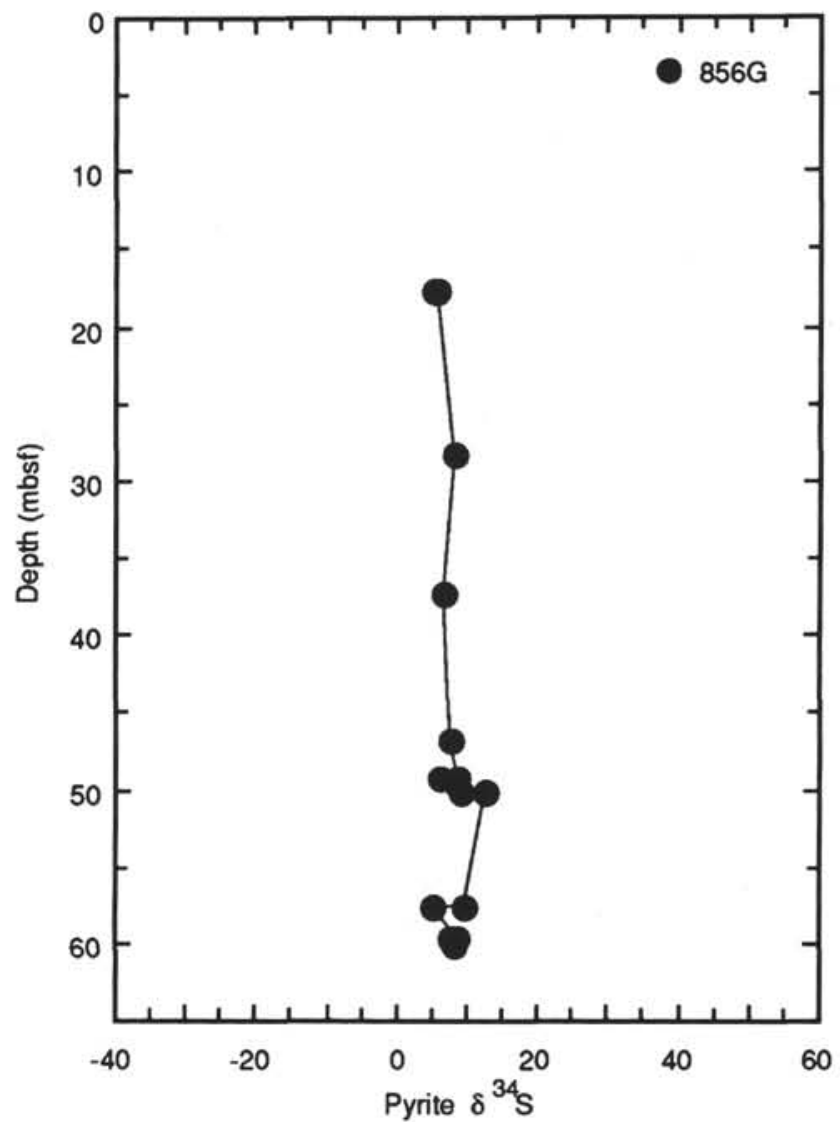

Figure 4. Sulfur isotope value of pyrite vs. depth for Hole 856G.

lighter value is considered to be more reliable and is close to the value of pyrite in the same sediment interval $(-12.0 \%)$. The sulfate analyzed is probably gypsum which formed by oxidation of pyrite. Pyrite in diabase from Hole $857 \mathrm{C}$ ranges in value from $-0.2 \%$ to $9.3 \%$. Petrographic observations indicate that all of the pyrite in the diabase is of hydrothermal origin and is not magmatic sulfide (Stakes and Franklin, this volume). The value of $-0.2 \%$ is from the chlorite-pyrite selvage of an epidote vein cutting diabase and the value of $9.3 \%$ is from a pyrite chlorite vein in diabase. Two samples of sphalerite from wairakite-bearing veins in diabase have values of $7.5 \%$ and $7.6 \%$ Pyrite in sediment from the section intruded by the diabase sills tends to be several per mil heavier than pyrite in the diabase.

\section{Hole $857 D$}

Pyrite sampled from Hole 857D includes hydrothermal pyrite in diabase sills and pyrite in sediment between diabase sills. Pyrite in sediment ranges in $\delta^{34} \mathrm{~S}$ value from $9.0 \%$ to $14.6 \%$ and is generally heavier than pyrite in adjacent diabase sills (Fig. 6). One sample of pyrrhotite in sediment has a value of $8.3 \%$, $1.3 \%$ lighter than coexisting pyrite. The temperature calculated by assuming isotopic equilibrium between the pair is $110^{\circ} \mathrm{C}$, suggesting that the assumption of equilibrium is not valid, as it is below the present temperature at this depth. Pyrrhotite from a quartz-pyrrhotite vein has a value of $8.7 \%$. Pyrite in diabase sills ranges from 7.2\% to $12.0 \%$. Pyrite in veins cutting diabase falls within this range. Pyrrhotite from a wairakite-quartz vein cutting diabase has a value of $8.2 \%$; sphalerite from wairakitebearing veins gives values of $7.7 \%$ and $8.6 \%$.

\section{Site $\mathbf{8 5 8}$}

Drilling at Site 858 consisted of a transect of holes from the margin to the center of an active hydrothermal field. Approximately 20 active 


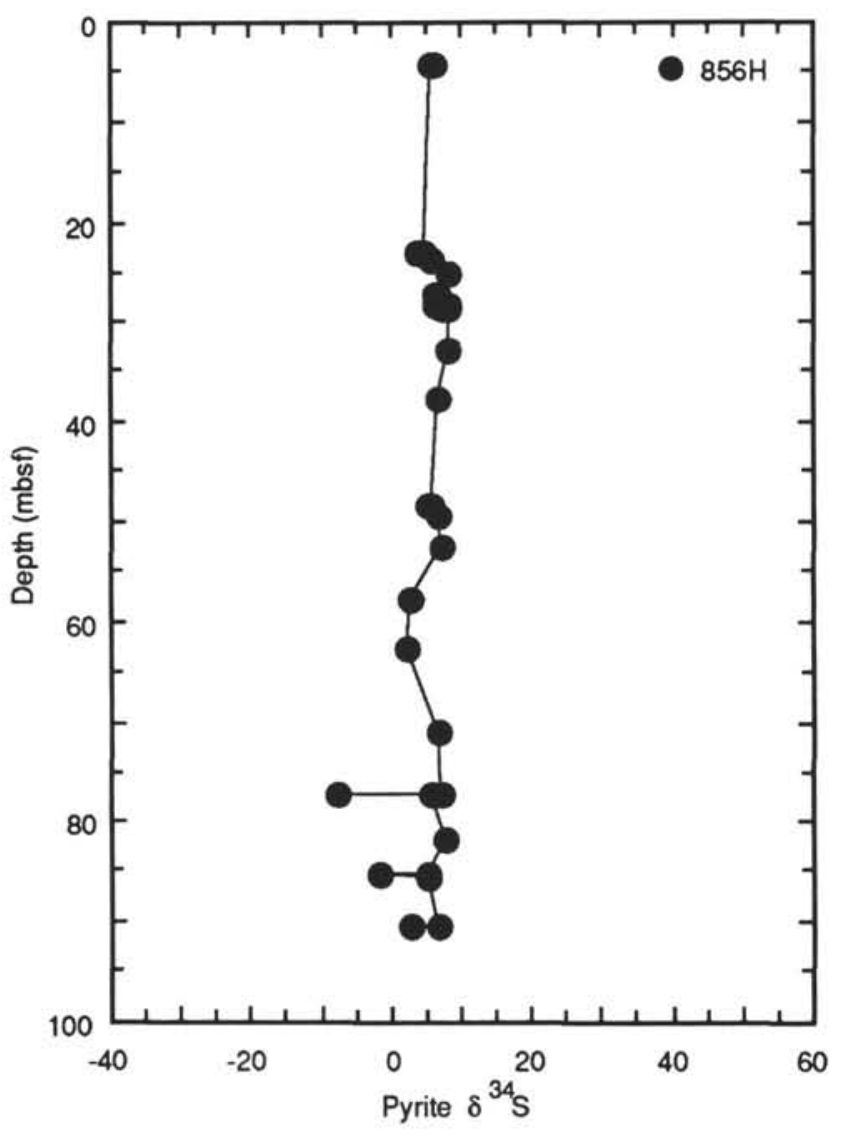

Figure 5. Sulfur isotope value of pyrite vs. depth for Hole $856 \mathrm{H}$.

hydrothermal vents have been mapped in this area. Hydrothermal fluid at temperatures up to $276^{\circ} \mathrm{C}$ vents from anhydrite chimneys which contain only minor amounts of sulfide (Davis, Mottl, Fisher, et al., 1992). Pore-water sulfate profiles in all of the Site 858 holes cannot be interpreted simply in terms of bacterial sulfate reduction because the high temperature of the sediment has resulted in precipitation anhydrite, some of which can redissolve during core retrieval and processing (Davis, Mottl, Fisher, et al., 1992). Similarly, sulfur contents of sediments from Site 858 reported in Davis, Mottl, Fisher, et al. (1992) cannot be interpreted in terms of pyrite content because of the presence of abundant anhydrite in many sediment samples. Percent sulfur reported in Table 1 of this report does represent the sulfide-sulfur, and not sulfate-sulfur, content of the sediment samples.

\section{Hole 858A}

Hole $858 \mathrm{~A}$ is located about $200 \mathrm{~m}$ west of the center of the active vent field and was APC-XCB cored to a depth $339.1 \mathrm{~m}$ in turbiditic and hemipelagic sediment and metasediment. Pyrite $\delta^{34} \mathrm{~S}$ values range from $-32.4 \%$ to $28.9 \%$ (Fig. 7). A downhole increase in $\delta^{34} \mathrm{~S}$ values is not as readily apparent in Hole $858 \mathrm{~A}$ as in holes further from the hydrothermal center. However, samples from below $200 \mathrm{mbsf}$ do have relatively constant sulfur isotope values near $9 \%$, similar to deep samples from other Middle Valley sites. Anhydrite and gypsum that occur disseminated in sediment, cementing sediment, or as veins have $\delta^{34} \mathrm{~S}$ values of $21.3 \%$ to $28.3 \%$. The lowest values are near the value of seawater sulfate, but most samples are enriched in ${ }^{34} \mathrm{~S}$ by several per mil.

\section{Hole $858 B$}

Hole $858 \mathrm{~B}$ was hydraulically piston cored $20 \mathrm{~m}$ away from an active hydrothermal vent. Sediment recovered from this short hole

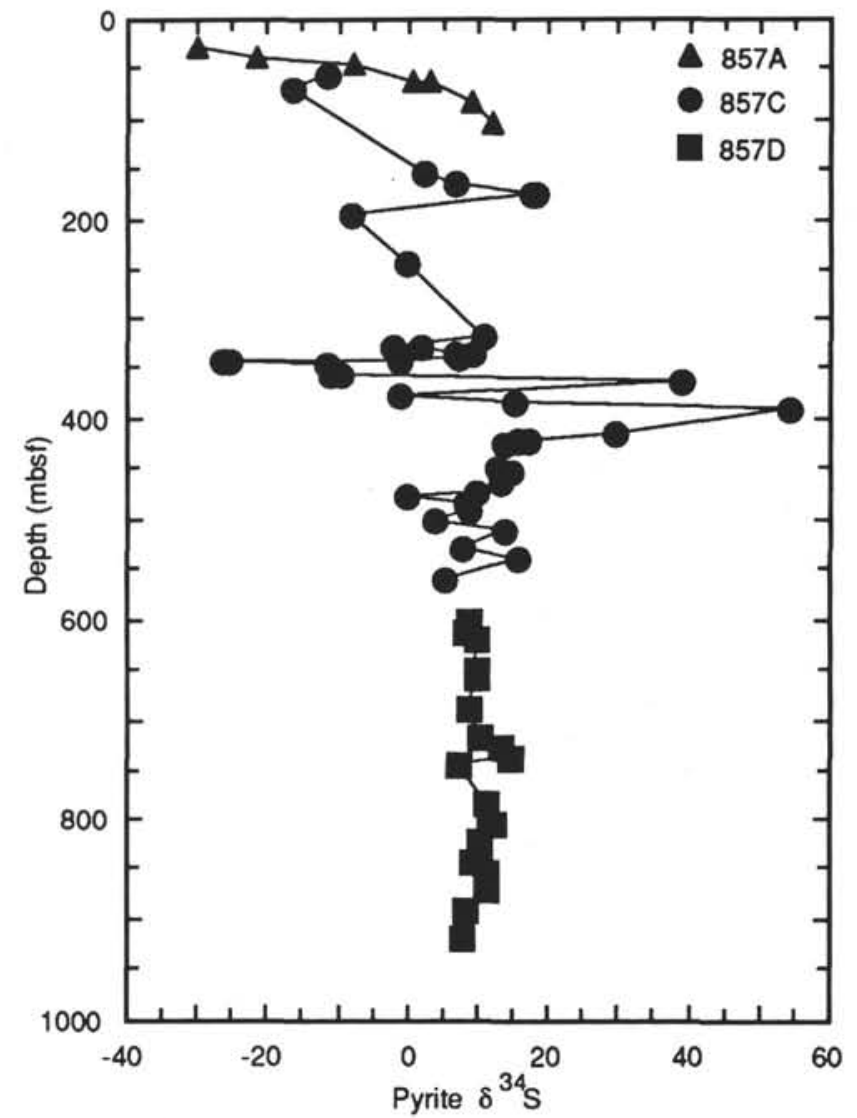

Figure 6. Sulfur isotope value of pyrite vs. depth for Holes 857A, 857C, and 857D.

was highly altered by magnesium metasomatism and contained abundant pyrite and anhydrite, including an interval containing semimassive sulfide at approximately $10-12 \mathrm{mbsf}$. Pyrite $\delta^{34} \mathrm{~S}$ values range from $-15.3 \%$ to $4.5 \%$ (Fig. 8). Samples of semimassive sulfide have values of $1.7 \%$ to $4.5 \%$. Anhydrite in the altered sediment has values of $23.7 \%$ and $26.3 \%$. One sample of barite is highly enriched in ${ }^{34} \mathrm{~S}$ with a value of $44.6 \%$.

\section{Hole $858 \mathrm{C}$}

Hole $858 \mathrm{C}$ was APC-XCB cored to a depth of $93.1 \mathrm{~m}$. This hole is located within $200 \mathrm{~m}$ of both Hole $858 \mathrm{~A}$ and Hole $858 \mathrm{~B}$ and is approximately $100 \mathrm{~m}$ away from the Dead Dog mound hydrothermal vents. Pyrite in sediment from this hole has $\delta^{34} \mathrm{~S}$ values of $-9.2 \%$ to $15.2 \%$ (Fig. 8). Pyrite sulfur isotope values are less variable downhole than in Hole 858A. The deepest samples have values near $8 \%$ except for the sample from near the bottom of the hole at $84.46 \mathrm{mbsf}$, which has the lowest value in the hole $(-9.2 \%)$. Anhydrite from the middle section of this hole has values between $22.3 \%$ and $23.8 \%$, similar to those in Hole 858A. However, three samples from the top $30 \mathrm{~m}$ of the hole and the deepest sample in the hole have values below that of seawater sulfate and require input of a lighter source of sulfur, such as $\mathrm{H}_{2} \mathrm{~S}$ or pyrite that has been oxidized to sulfate.

\section{Holes $858 D, 858 G$, and $858 F$}

Holes $858 \mathrm{D}, 858 \mathrm{G}$, and $858 \mathrm{~F}$ were drilled in the center of the heat flow anomaly that defines the active vent field, but are not immediately adjacent to any active hydrothermal vents. Hole $858 \mathrm{D}$ was drilled by APC-XCB to $40.7 \mathrm{~m}$, Hole $858 \mathrm{G}$ was rotary cored to 296.9 $\mathrm{m}$, and Hole $858 \mathrm{~F}$ was rotary cored from $276.5 \mathrm{~m}$ to $432.6 \mathrm{~m}$. Both of 


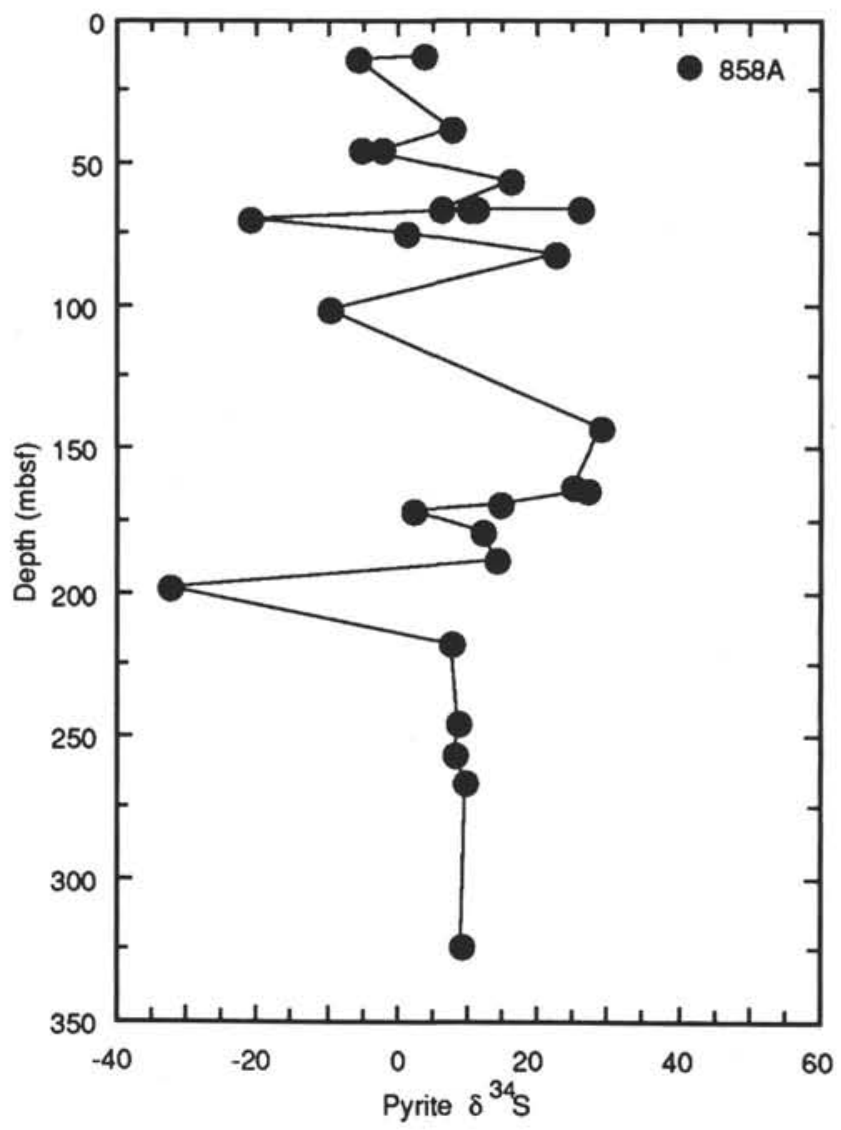

Figure 7. Sulfur isotope value of pyrite vs. depth for Hole 858A.

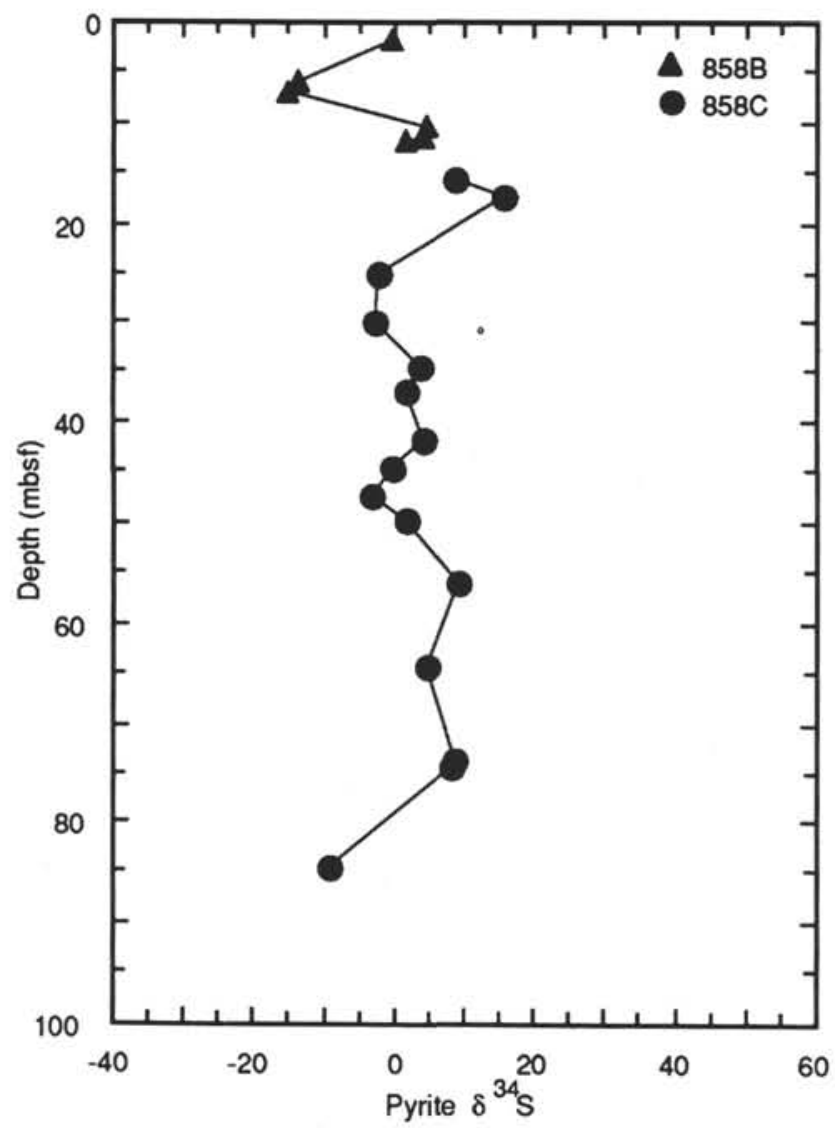

Figure 8 . Sulfur isotope value of pyrite vs. depth for Holes $858 \mathrm{~B}$ and $858 \mathrm{C}$.

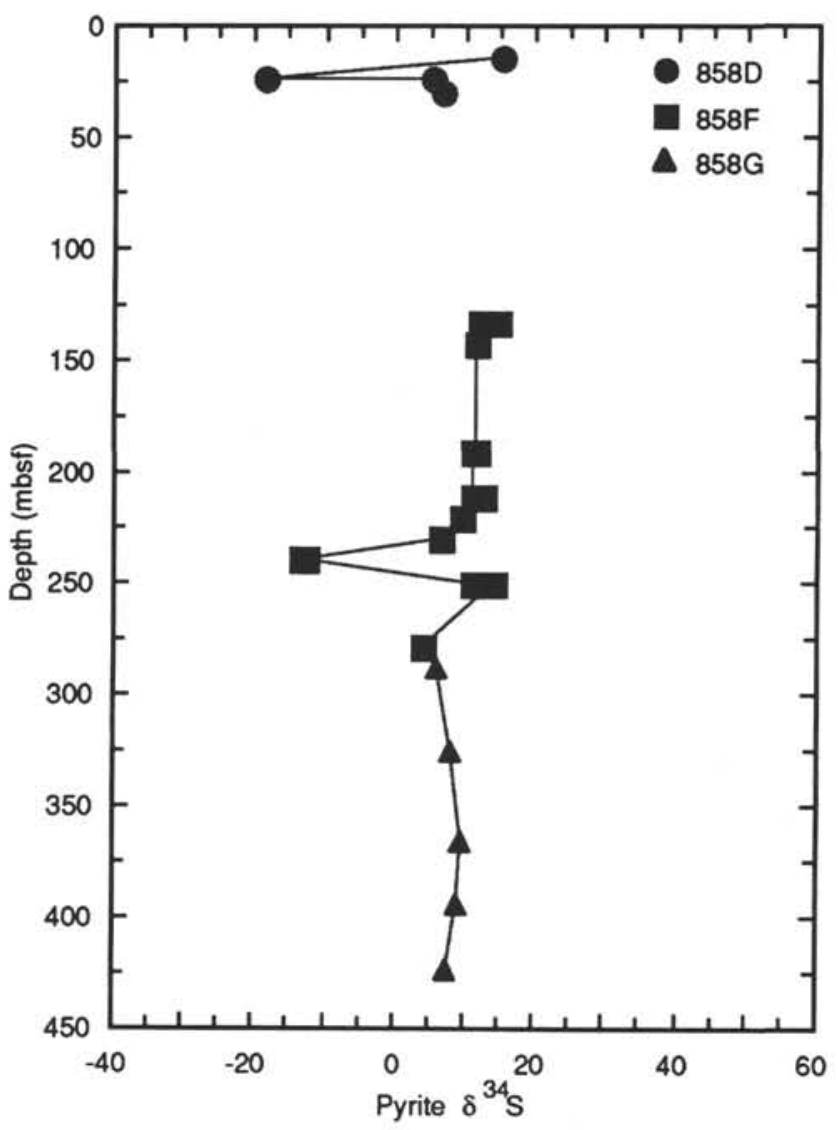

Figure 9. Sulfur isotope value of pyrite vs. depth for Holes $858 \mathrm{D}, 858 \mathrm{~F}$, and $858 \mathrm{G}$.
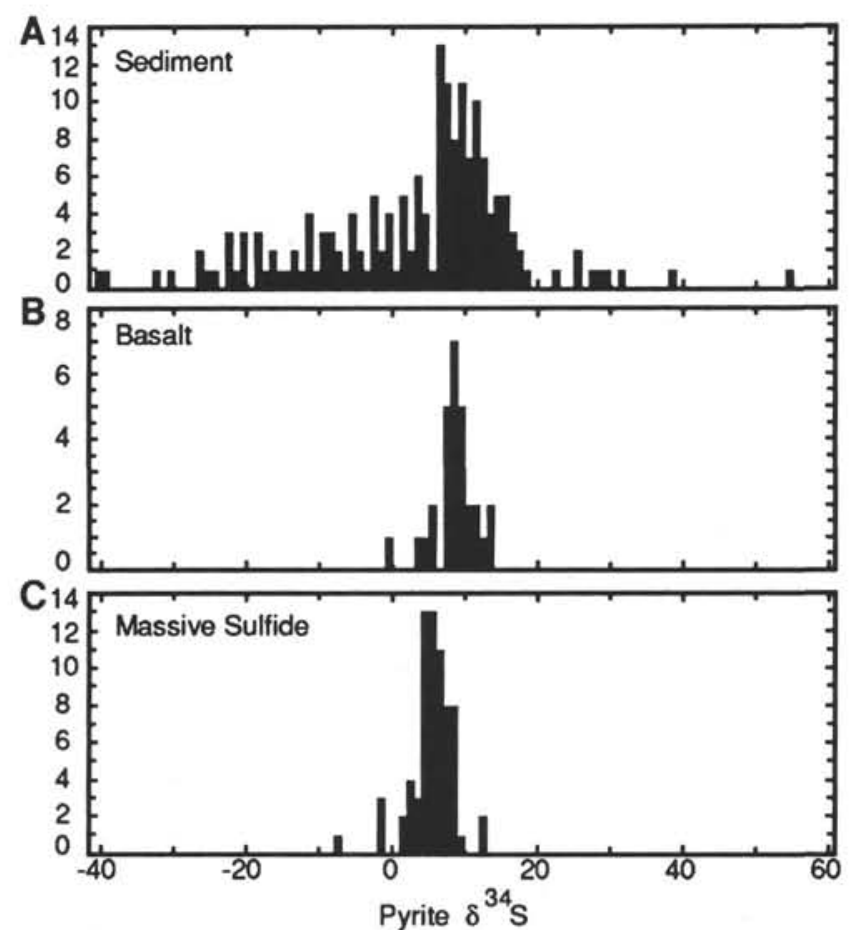

Figure 10. Histograms comparing the sulfur isotope values of pyrite in (A) sediment and metasedimentary rock, (B) basalt and diabase, and (C) massive, semimassive, and clastic sulfide. 
the rotary cored holes had low core recovery. Basalt was recovered in Holes $858 \mathrm{G}$ and $858 \mathrm{~F}$ below $249 \mathrm{mbsf}$. Pyrite in sediment from Hole $858 \mathrm{D}$ ranges in $\delta^{34} \mathrm{~S}$ from $-18.7 \%$ to $15.1 \%$ (Fig. 9). Anhydrite has values of $23.2 \%$ and $23.5 \%$. The deeper sedimentary section sampled by Hole $858 \mathrm{~F}$ contains pyrite with $\delta^{34} \mathrm{~S}$ values ranging from $-13.2 \%$ to $14.7 \%$, but most samples have a narrow range, from $11 \%$ to $12 \%$ (Fig. 9). Two samples of anhydrite have $\delta^{34} \mathrm{~S}$ values slightly higher than seawater sulfate $(22.1 \%$ and $23.4 \%$ ), but one sample has a low value of $11.4 \%$, similar to disseminated pyrite in the same sample $(11.0 \%)$. Pyrite in quartz-wairakite veins in basalt has $\delta^{34} \mathrm{~S}$ values of $13.3 \%$ and $13.8 \%$. Disseminated pyrite in basalt has a value of $4.0 \%$. All of the samples from Hole $858 \mathrm{G}$ are basalt or metabasalt. Pyrite disseminated in basalt has values of $7.3 \%$ to $9.7 \%$; pyrite in chlorite veins cutting basalt has values of $5.8 \%$ and $8.1 \%$ (Fig. 9). One sample of pyrrhotite in basalt has a value of $10.3 \%$. An anhydrite vein cutting basalt has a $\delta^{34} \mathrm{~S}$ value of $22.5 \%$, slightly higher than seawater sulfate.

\section{SUMMARY OF DATA}

Near-surface sediments generally have pyrite with isotopically light sulfur typical of diagenetic sulfide produced by sulfate-reducing bacteria. Some isotopically heavy pyrite with $\delta^{34} \mathrm{~S}$ values greater than seawater sulfate is present in the sediment, requiring high degrees of biogenic(?) reduction of sulfate. Earlier diagenetic pyrite formed in near-surface sediment is overprinted, either with depth or by proximity to the center of the hydrothermal field, by isotopically heavy ( $6 \%$ to $12 \%$ ) pyrite of hydrothermal origin. Anhydrite occurs in the hotter sections of the sediment pile that underlies the hydrothermal field and has isotopic values that tend to be several per mil higher than seawater sulfate, consistent with precipitation from recharging seawater that has undergone isotopic fractionation by preferential extraction of ${ }^{32} \mathrm{~S}$ during sulfate reduction. Sulfide in altered igneous rocks is also isotopically heavy $\left(\delta^{34} \mathrm{~S}\right.$ approximately $8 \%$; Fig. 10). Thus, primary basaltic sulfide $(0.1 \%$, Sakai et al., 1984) is not the sole source of sulfur for the hydrothermal system; a heavier source of sulfur such as reduced seawater sulfate is required. Massive, semimassive, and clastic sul- fides have a relatively narrow range of $\delta^{34} \mathrm{~S}$ values near $6 \%$, similar to hydrothermal sulfides in both sedimentary and basaltic rock that underlies the deposit.

\section{ACKNOWLEDGMENTS}

This study was supported by the Gilbert Research Fellowship Program of the U.S. Geological Survey, for which the author is deeply grateful. Doug White of the Water Resources Division of the U.S. Geological Survey in Menlo Park is thanked for access to and assistance with mass spectrometry.

\section{REFERENCES}

Breit, G.N., Simmons, E.G., and Goldhaber, M.B., 1985. Dissolution of barite for the analysis of strontium isotopes and other chemical and isotopic variations using aqueous sodium carbonate. Chem. Geol., 52:333-336.

Canfield, D.E., Raiswell, R., Westrich, J.T., Reaves, C.M., and Berner, R.A., 1986. The use of chromium reduction in the analysis of reduced inorganic sulfur in sediments and shale. Chem. Geol., 54:149-155.

Davis, E.E., Mottl, M.J., Fisher, A.T., et al,, 1992. Proc. ODP, Init. Repts., 139: College Station, TX (Ocean Drilling Program).

Rees, C.E., 1978. Sulphur isotope measurements using $\mathrm{SO}_{2}$ and $\mathrm{SF}_{6}$. Geochim. Cosmochim. Acta, 42:383-389.

Rees, C.E., Jenkins, W.J., and Monster, J., 1978. The sulphur isotopic composition of ocean water sulphate. Geochim. Cosmochim. Acta, 42:377-381.

Sakai, H., Des Marais, D.J., Ueda, A., and Moore, J.G., 1984. Concentrations and isotope ratios of carbon, nitrogen and sulfur in ocean-floor basalts. Geochim. Cosmochim. Acta, 48:2433-2441. Abbreviations for names of organizations and publications in ODP reference lists follow
the style given in Chemical Abstracts Service Source Index (published by American
Chemical Society).

Date of initial receipt: 14 June 1993

Date of acceptance: 21 July 1993

Ms 139SR-226 